\title{
Clinical Outcomes of Metastatic Breast Cancer in Patients Having Both Pseudocirrhosis and Portal Hypertension with Evident Varices
}

\section{Wei-Li Ma}

National Taiwan University Hospital

\section{Dwan-Ying Chang}

National Taiwan University Hospital

Ching-Hung Lin

National Taiwan University Cancer Center Hospital

Kao-Lang Liu

National Taiwan University Hospital

Po-Chin Liang

National Taiwan University Hospital

Huang-Chun Lien

National Taiwan University Hospital

Chan-Chuan Hu

National Taiwan University Cancer Center

Ling-Yun Huang

National Taiwan University Hospital

Yi-Chun Yeh

National Taiwan University Hospital

Yen-Shen Lu ( $\nabla$ yslu@ntu.edu.tw )

National Taiwan University Hospital

\section{Research Article}

Keywords: metastatic breast cancer, liver, pseudocirrhosis, portal hypertension

Posted Date: November 11th, 2021

DOI: https://doi.org/10.21203/rs.3.rs-1039701/v1

License: (c) (1) This work is licensed under a Creative Commons Attribution 4.0 International License.

Read Full License 


\section{Abstract}

Background: Pseudocirrhosis is an imaging finding of malignancies with liver metastasis with or without clinical liver cirrhosis-related complications such as portal hypertension (pHTN). This study compared the outcomes of metastatic breast cancer in patients with imaging-diagnosed pseudocirrhosis with or without pHTN.

Methods: The medical records from patients with metastatic breast cancer and pseudocirrhosis between 2005 and 2017 were retrospectively analyzed. Clinical pHTN was defined based on endoscopic evidence of esophageal or gastric varices.

Results: Among 106 patients with pseudocirrhosis, 33 (31\%) had de novo stage IV disease, and 66 (62\%) had hormone receptor (HR) - positive and human epidermal growth factor receptor 2 (HER2)-negative breast cancer. In total, 81 (76\%) had initial metastases in both hepatic lobes, 91 (86\%) had 4 or more liver metastases, and 32 (30\%) had pHTN. The median overall survival (OS) was 5 and 13 months in patients with and without pHTN, respectively $(p=.002)$. The median OS in patients with HER2-positive, HRpositive/HER2-negative, and triple-negative breast cancer was 16,9 , and 2 months, respectively ( $p=$ .001). Patients with pHTN generally had cirrhotic complications, including gastrointestinal bleeding, hyperbilirubinemia, hyperammonemia, and coagulopathy. Despite their challenging clinical conditions, 7 patients with pHTN had OS exceeding 1 year. In multivariate analysis, pHTN ( $p=.007)$ and triple-negative breast cancer $(p=.013)$ were associated with poor OS.

Conclusions: For patients with pseudocirrhosis, clinical pHTN was associated with liver cirrhosis-related complications and shorter median OS. A few patients with pHTN had prolonged OS with effective systemic treatment and aggressive supportive care.

\section{Background}

Metastasis is the primary cause of breast cancer mortality [1]. Compared with bone or lung metastases, liver metastases usually signify a severe disease burden and worse prognosis [2]. Hepar lobatum carcinomatosum, metastatic carcinomatous liver cirrhosis, and pseudocirrhosis are terms used in radiologic imaging to describe rare hepatic morphological abnormalities that resemble liver cirrhosis but without the manifestation of chronic liver disease [3-5]. Characteristic features include lobular hepatic contour, segmental volume loss, and caudate lobe enlargement [6]. Although pseudocirrhosis is most often associated with breast cancer, it has also been reported in malignancies of the gastrointestinal (GI) tract, medullary thyroid, and ovary [7-12]. Most published studies are individual case reports, and larger case series are scarce [13-18].

Portal hypertension ( $\mathrm{pHTN}$ ) results in major complications that mark the transition from compensated to decompensated cirrhosis [19]; these include ascites, splenomegaly, gastroesophageal varices bleeding, spontaneous bacterial peritonitis, and hepatorenal syndrome [20]. Secondary pHTN has been reported in 
patients with cancer and attributed to various causes, including liver metastasis, chemotherapy-induced hepatic sinusoidal obstruction, or radiotherapy localized to the liver [21-25].

The term "pseudocirrhosis" may have misled researchers and clinicians to overlook the clinical importance of pHTN in affected patients, and investigations of the impact of pseudocirrhosis on survival has been limited. In clinical practice, up to one-quarter of patients have both "pseudocirrhotic" imaging results and concurrent clinically compromised liver function with typical complications associated with pHTN [18]. Therefore, we conducted this retrospective study to investigate how the presence of pHTN in patients with pseudocirrhosis affects the clinical outcomes of metastatic breast cancer.

\section{Methods}

Patient Selection, Stratification, And Analytic Data

The medical records from patients with metastatic breast cancer and imaging-diagnosed pseudocirrhosis between 2005 and 2017 were retrospectively analyzed. Among 164 potentially eligible patients, 58 were excluded: 32 had malignancies other than breast cancer, and 26 had incomplete clinical data or imaging findings. The remaining 106 patients were included in the analysis. Radiologists coded pseudocirrhosis based on the presence of lobular hepatic contours, segmental volume loss, and caudate lobe enlargement (Fig. 1). Patients with advanced breast cancer may have ascites (including malignant ascites), cachexia-related hypoalbuminemia, or splenomegaly without confirmed pHTN; therefore, we used a stringent criterion to define clinical pHTN, namely the presence of esophageal or gastric varices on esophagogastroduodenoscopy (EGD). Eligible patients were divided into groups with or without EGDdefined pHTN. Their ages, breast cancer stages, pathology subtypes, and viral hepatitis status upon breast cancer diagnosis were retrieved from medical records. The initial numbers of metastases in liver lobes, other concurrent visceral metastases present at pseudocirrhosis development, and the interval from stage IV disease to documented pseudocirrhosis were also excerpted for analysis. In addition, systemic chemotherapy regimens and the conventional complications of pHTN and decompensated cirrhosis after pseudocirrhosis diagnosis were reviewed, including GI bleeding, thrombocytopenia, hyperbilirubinemia, coagulopathy, and hyperammonemia. Thrombocytopenia was defined as platelet count of $<100,000 / \mu \mathrm{L}$, hyperbilirubinemia as total bilirubin $>1.5$ times the upper limit of normal, coagulopathy as International Normalized Ratio (INR) $>1.5$ times the upper limit of normal, and hyperammonemia as blood ammonia $>50 \mu \mathrm{mol} / \mathrm{L}$. Most patients underwent computed tomography assessments every 3 months as per the Taiwan National Health Insurance regulations. Overall survival (OS) was defined as the interval from the date of pseudocirrhosis diagnosis until the date of death from any cause.

The National Taiwan University Hospital Research Ethics Committee (NTUHREC) approved this retrospective study and the waived informed consent (NTUHREC No. 201806028RINC). The medical charts were retrospectively reviewed and the all process was carried out in accordance with the regulations of Helsinki. 


\section{Statistical Analysis}

The categorical variables of patients with and without pHTN were compared using Fisher's exact test. Continuous variables and medians were compared using the Mann-Whitney U test. OS was estimated using the Kaplan-Meier method. Univariate and multivariate Cox proportional hazard models with timedependent covariates were used to explore associations among pHTN, concurrent lung or brain metastases, and other factors with potential prognostic value for determining OS. The $p$ value of $<.05$ was considered statistically significant.

\section{Results}

\section{Patient Characteristics}

Table 1 shows the clinical characteristics of patients with pseudocirrhosis. As revealed by EGD, 32 patients had clinical pHTN and 74 patients were without pHTN. Patient and tumor characteristics between the 2 groups were generally similar. Overall, the median age was 54 years, 73 patients $(69 \%)$ had recurrent metastatic breast cancer, $66(62 \%)$ had hormone receptor-positive and human epidermal growth factor receptor 2-negative (HR+/HER2-; luminal-type) disease, 32 (30\%) had HER2+ disease, and 8 (8\%) had triple-negative disease. Serologically, 9 patients were hepatitis B virus surface antigen (HBsAg) positive, and 1 tested positive for anti-hepatitis $\mathrm{C}$ virus antibody (anti-HCV). Moreover, 7 of 9 patients positive for HBsAg received antiviral therapy during systemic anticancer treatment. Of the 9 patients positive for HBsAg, 3 had pHTN, whereas the remaining 6, including the only patient who was antiHCV(+), did not have pHTN. Moreover, 81 (76\%) patients had liver metastases in both lobes, and 91 (86\%) had 4 or more liver metastases preceding pseudocirrhosis development. In the study population, 43 (41\%) and $23(22 \%)$ patients had concurrent lung metastases and brain metastasis, respectively. The median time from the diagnosis of metastatic breast cancer to the development of pseudocirrhosis was 34 months in patients with pHTN and 26 months in those without $(p=.315)$. All patients received considerable pretreatment before pseudocirrhosis was documented (Supplementary Table 1). The majority $(86 \%)$ of the patients had received at least 4 lines of chemotherapy since metastasis, and 73 (69\%) had received 5 or more lines. Among the 66 patients with HR+/HER2- breast cancer, 45 (68\%) had received at least 2 lines of endocrine therapy for metastatic disease.

\section{Clinical Courses and Survival outcomes after Diagnosis of Pseudocirrhosis}

Table 2 shows the systemic chemotherapy regimens and complications of $\mathrm{pHTN} /$ hepatic decompensation after the diagnosis of pseudocirrhosis. Regardless of the presence of pHTN, the most frequently prescribed drugs were fluorouracil ( $5-\mathrm{FU})$ and platinum, with these drugs were prescribed for $66 \%$ and $47 \%$ of patients in the pHTN group as well as $52 \%$ and $46 \%$ of patients in the non-pHTN group, respectively. Forty-five patients without pHTN received paclitaxel and docetaxel (61\%), compared with 10 patients with pHTN (31\%; $\mathrm{p}=.005)$. In addition, 21 (28\%) patients without pHTN and $3(9 \%)$ patients with pHTN received doxorubicin $(\mathrm{p}=.042)$. Patients with $\mathrm{pHTN}$ experienced more manifestations implying hepatic decompensation or complications of $\mathrm{pHTN}$. The incidence rate ranged from the lowest of $59 \%$ for 
hyperammonemia to the highest of $91 \%$ for thrombocytopenia; $72 \%$ of patients experienced GI bleeding, and $84 \%$ had received blood transfusion. For patients without pHTN, the most common complication was thrombocytopenia (55\%), and only 6 (8\%) patients developed GI bleeding. Patients with pHTN had a median OS of only 5 months compared with 13 months in those without pHTN $(p=.002$; Fig. 2$)$. The median OS of patients with HER2-positive, luminal-type, and triple-negative breast cancer was 16, 9, and 2 months, respectively ( $p=.001 ; \mathrm{Fig} .3$ ). Generally, the prognosis of patients with pseudocirrhosis and pHTN is poor; nevertheless, in the present study, with proper treatment and supportive care, $7(22 \%)$ patients had OS exceeding 1 year. Among these patients, 5 hadHR+/HER2- disease, and 2 had HER2+ disease.

Univariate and Multivariate Analyses of OS

Univariate analysis of OS was performed to assess the prognostic impact of age, $\mathrm{pHTN}$, pathology subtype, de novo or recurrent metastastic disease, concurrent lung metastasis, and concurrent brain metastasis. In a univariate analysis, pHTN (Hazard ratio: 2.16 [95\% confidence interval $\{\mathrm{Cl}\}: 1.33-3.53$ ], $p$ $=.002$ ), triple-negative breast cancer (Hazard ratio: 2.91 [95\% Cl: 1.19-7.08], $p=.019$ ), and concurrent metastasis to the brain (Hazard ratio: 1.83 [95\% Cl: 0.99-3.38], $p=.054$ ) were associated with poor survival. Only pHTN (Hazard ratio: 2.09 [95\% Cl: 1.22-3.57], $p=.007$ ) and triple-negative breast cancer (Hazard ratio: 3.15 [95\% Cl: 1.27-7.81], $p=.013$ ) were associated poor OS in a multivariate analysis (Table 3).

\section{Discussion}

In this single-institution retrospective analysis, we identified 106 metastatic breast cancer patients with pseudocirrhosis on radiology reports within a 13-year interval. To our knowledge, this is the largest series to date; because pseudocirrhosis was diagnosed by a random radiologist, we postulated that these image changes would be conspicuous rather than subtle. In other words, the actuarial patient number may be higher if a systemic image review is performed. Pseudocirrhosis can be complicated by pHTN[13,15,16,18,26-33]. In contrast to earlier studies that have used imaging criteria to define pHTN, we chose a stringent criterion to define pHTN: the presence of varices on EGD. Despite the use of this criterion, up to $30 \%$ of patients $(n=32)$ were identified as having pHTN, and this too is the largest case series thus far. Oliai et alretrospectively analyzed a consecutive cohort at the University of California Los Angeles and reported that 37 of 199 consecutively treated patients with metastatic breast cancer developed pseudocirrhosis, and of patients with liver metastases, 55\% developed pseudocirrhosis [17]. According to that report, pseudocirrhosis in metastatic breast cancers is not rare. However, the term "pseudocirrhosis" is sometimes misleading because some of these patients have hepatic decompensation.

Although our study did not focus on the risk factors and etiologies of pseudocirrhosis, our findings are consistent with those in a series reported by Oliai et al [17]. In their series, they found that pseudocirrhosis does not occur in the absence of liver metastases, and those who developed pseudocirrhosis had 
received more lines of systemic treatments; they thus concluded that higher cumulative exposure to systemic treatment may be causative in contrast to the previous assumption that pseudocirrhosis is an adverse effect of a particular therapeutic agent. In our cohort, every patient had preceding liver metastases, $76 \%$ had bilateral lobe involvement, and $61 \%$ had at least 5 hepatic nodules upon liver metastases. In the entire cohort, $69 \%$ had received 5 or more lines of chemotherapies, and among patients who were $\mathrm{HR}+, 68 \%$ had received 2 or more lines of endocrine therapy. The median time from the diagnosis of metastatic disease to the development of pseudocirrhosis was $27(0-108)$ months, which is in line with the postulation that pseudocirrhosis usually affects patients with considerable pretreatment and is a late event.

In the literature, limited data on the histology change of pseudocrirrhosis are available. In our study, 1 patient had undergone liver biopsy, and the pathology finding was fatty change and fibrosis without malignant cells (Fig. 4). The finding was different from those of Young et aland Diamond et al, who have suggested nodular regeneration hyperplasia or intravascular tumor infiltration with extensive stromal fibrosis and compression of the portal triad vasculature, respectively [13,34]. The pathogenesis may consist of a spectrum and may change along the disease course, which warrants comprehensive research.

Because we defined pHTN by the presence of esophageal or gastric varices in this study, it was unsurprising to find that in addition to triple-negative disease, pHTN is also independently associated with poor OS. Patients with pHTN had significantly more complications of hepatic decompensation, including hyperbilirubinemia, thrombocytopenia, coagulopathy, and hyperammonemia. Moreover, variceal bleeding can be lethal. We summarize data on 25 breast cancer patients with pseudocirrhosis complicated by esophageal or gastric varices in previous case reports and series in Table $4[13,15,16,18,26-33]$. Most of these patients had HR+/HER2- disease, and OS was approximately 1 month from the diagnosis of esophageal or gastric varices. In our 32 patients, 23 had GI bleeding episodes, and 11 deaths were associated with variceal bleeding. The median OS of 5 months in this study is congruent with the OS of approximately 1 month reported in previous studies[16,26,30,31]. Poor liver reserve hampered further anticancer treatment. Except for platinum and infusional 5-FU, almost every chemotherapy drug commonly used for breast cancer requires substantial dose modification or is even contraindicated in patients with hepatic dysfunction. This explains why cisplatin and infusional 5FU were most frequently prescribed after pseudocirrhosis was documented in our study. Furthermore, patients with pHTN may have concomitant ascites, nutrition problems, and resultant poor functional performance. Although managing these patients is challenging, 7 patients with pseudocirrhosis and pHTN had OS exceeding 1 year. Moreover, 5 patients had HR+/HER2-disease, and 2 had HER2+ disease. We believe that effective systemic treatment and aggressive supportive care can prolong the survival of such patients.

The limitations of this study include its retrospective design and the heterogeneous patient sample with different breast cancer subtypes and diverse treatment protocols. The study data were recorded between 2005 and 2017; anticancer drugs approved after 2017 might have improved outcomes. This study 
evaluated the clinical characteristics and outcomes of patients with breast cancer who had pseudocirrhosis with and without pHTN. Additional studies are warranted to investigate other prognostic factors and chemotherapy dosage regimens as well as the detailed pathogenesis of pHTN in patients with pseudocirrhosis.

\section{Conclusion}

In our study cohort, pseudocirrhosis mostly developed in patients with luminal-type breast cancer initially diagnosed at an early stage, but these patients had recurrence with multiple liver metastases. Considerable pretreatment ( $80 \%$ had received 5 or more chemotherapy lines) suggests that pseudocirrhosis is a relatively late event. Patients with and without pHTN, as defined by EGD-documented varices, had significantly shorter OS; nevertheless, a few patients survived for longer than 1 year when given aggressive anticancer therapy and intensive supportive care.

\section{Abbreviations}

pHTN: portal hypertension; HR: hormone receptor; HER2: human epidermal growth factor receptor 2; OS: overall survival; GI: gastrointestinal; EGD: esophagogastroduodenoscopy; INR: International Normalized Ratio; HBsAg: hepatitis B virus surface antigen; anti-HCV: anti-hepatitis $\mathrm{C}$ virus antibody; 5 -FU: fluorouracil; Cl: confidence interval

\section{Declarations}

\section{Ethics approval and consent to participate}

The National Taiwan University Hospital Research Ethics Committee (NTUHREC) approved this retrospective study and the waived informed consent (NTUHREC No. 201806028RINC). The medical charts were retrospectively reviewed and the all process was carried out in accordance with the regulations of Helsinki.

\section{Consent for publication}

Not applicable.

\section{Availability of data and material}

The datasets used and analyzed during the current study are available from the corresponding author on reasonable request.

\section{Competing interests}

The authors have no conflicts of interest to declare. 


\section{Funding}

This study was supported by Ministry of Health and Welfare MOHW110-TDU-B-211-144017. The funding body had no role in the study design, data collection, analysisand interpretation, and in writing the manuscript.

\section{Authors' contributions}

W.L.M. and D.Y.C. were responsible for literature collection, data management and interpretation, and manuscript writing. W.L.M., D.Y.C., C.H.L., K.L.L., P.C.L., and H.C.L contributed to patient care and clinical data. C.C.H., L.Y.H. and Y.C.Y. were responsible for data management and interpretation, and statistical analysis. Y.S.L. treated patients; planned, designed, and coordinated the study throughout the study period; and wrote the manuscript.All authors read and approvedthe final manuscript.

\section{Acknowledgements}

The authors thank the Integrated Medical Database, National Taiwan University Hospital, and Department of Medical Research, National Taiwan University Hospital, for sharing patient information. We also thank our colleagues at the Department of Medical Research, National Taiwan University Hospital, for providing consultation.Dr. David Neil, Ph.D., of the Dr. Word company (Taipei, Taiwan) provided the professional English editing service.

\section{References}

1. Scully OJ, Bay BH, Yip G, Yu Y. Breast cancer metastasis. Cancer Genomics Proteomics. 2012;9:311320.

2. Li J, Zhu H, Sun L, Xu W, Wang X. Prognostic value of site-specific metastases in lung cancer: A population based study. J Cancer. 2019;10:3079-3086.

3. Honma K. Heparlobatumcarcinomatosum due to metastatic breast carcinoma. Virchows Arch A PatholAnatHistopathol. 1987;410:465-469.

4. Micolonghi T, Pineda E, Stanley MM. Metastatic carcinomatous cirrhosis of the liver; report of a case in which death followed hemorrhage from esophageal varices and hepatic coma. AMA Arch Pathol. 1958;65:56-62.

5. Chandrakar V, Isaacs C. Breast cancer-related pseudocirrhosis and esophageal varices. Breast J. 2005;11:301-302.

6. Jha P, Poder L, Wang ZJ, Westphalen AC, Yeh BM, Coakley FV. Radiologic mimics of cirrhosis. AJR Am J Roentgenol. 2010;194:993-999.

7. Kobashigawa C, Nakamoto M, Hokama A, Hirata T, Kinjo F, Fujita J. Pseudocirrhosis in metastatic esophageal cancer. South Med J. 2010;103:488-489.

8. Mitani S, Kadowaki S, Taniguchi H, Muto H, Muro K. Pseudocirrhosis in Gastric Cancer with Diffuse Liver Metastases after a Dramatic Response to Chemotherapy. Case Rep Oncol. 2016;9:106-111. 
9. Battisti S, Guida FM, Pagliara E, Tonini G, Zobel BB, Santini D. Pseudocirrhosis after anti-EGFR-based neoadjuvant therapy for hepatic metastasis from colon cancer: a different point of view. Clin Colorectal Cancer. 2014;13:e13-e15.

10. Kang SP, Taddei T, McLennan B, Lacy J. Pseudocirrhosis in a pancreatic cancer patient with liver metastases: a case report of complete resolution of pseudocirrhosis with an early recognition and management. World J Gastroenterol. 2008;14:1622-1624.

11. Harry BL, Smith ML, Burton JR Jr, Dasari A, Eckhardt SG, Diamond JR. Medullary thyroid cancer and pseudocirrhosis: case report and literature review. CurrOncol. 2012;19:e36-e41.

12. Zanazanian A, Kalani A, Tabibian JH. Pseudocirrhosis in a patient with new-onset dysphagia and history of ovarian carcinoma. ACG Case Rep J. 2018;5:e29.

13. Young ST, Paulson EK, Washington K, Gulliver DJ, Vredenburgh JJ, Baker ME. CT of the liver in patients with metastatic breast carcinoma treated by chemotherapy: findings simulating cirrhosis. AJR Am J Roentgenol. 1994;163:1385-1388.

14. Qayyum A, Lee GK, Yeh BM, Allen JN, Venook AP, Coakley FV. Frequency of hepatic contour abnormalities and signs of portal hypertension at CT in patients receiving chemotherapy for breast cancer metastatic to the liver. Clin Imaging. 2007;31:6-10.

15. Sonnenblick A, Appelbaum L, Peretz T. Liver failure on the background of pseudocirrhosis in patients with liver metastasis from breast cancer, who responded to treatment. Onkologie. 2011;34:199-201.

16. Adike A, Karlin N, Menias C, Carey EJ. Pseudocirrhosis: a case series and literature review. Case Rep Gastroenterol. 2016;10:381-391.

17. Oliai C, Douek ML, Rhoane C, Bhutada A, Ge PS, Runyon BA, et al. Clinical features of pseudocirrhosis in metastatic breast cancer. Breast Cancer Res Treat. 2019;177:409-417.

18. Engelman D, Moreau M, Lepida A, Zaouak Y, Paesmans M, Awada A. Metastatic breast cancer and pseudocirrhosis: an unknown clinical entity. ESMO Open. 2020;5:e000695.

19. D'Amico G, Garcia-Tsao G, Pagliaro L. Natural history and prognostic indicators of survival in cirrhosis: a systematic review of 118 studies. J Hepatol. 2006;44:217-231.

20. Berzigotti A. Advances and challenges in cirrhosis and portal hypertension. BMC Med. 2017;15:200.

21. Theophilidou E, Waraich N, Raza T, Agarwal PK. Liver metastases, a rare cause of portal hypertension and stoma bleeding. Brief review of literature. Int J Surg Case Rep. 2012;3:173-176.

22. Tisman G, MacDonald D, Shindell N, Reece E, Patel P, Honda N, et al. Oxaliplatin toxicity masquerading as recurrent colon cancer. J ClinOncol. 2004;22:3202-3204.

23. Rubbia-Brandt L, Audard V, Sartoretti P, Roth AD, Brezault C, Le Charpentier M, et al. Severe hepatic sinusoidal obstruction associated with oxaliplatin-based chemotherapy in patients with metastatic colorectal cancer. Ann Oncol. 2004;15:460-466.

24. Jakobs TF, Saleem S, Atassi B, Reda E, Lewandowski RJ, Yaghmai V, et al. Fibrosis, portal hypertension, and hepatic volume changes induced by intra-arterial radiotherapy with $90 y$ ttrium microspheres. Dig Dis Sci. 2008;53:2556-2563. 
25. Gutierrez L, Méndez S, Mitjavila M, Llop E, Salas C, Ruiz-Casado A. Noncirrhotic portal hypertension: An under-reported late adverse event of SIRT in metastatic colorectal cancer patients. J Cancer Res Ther. 2019;15:42-47.

26. Nascimento AB, Mitchell DG, Rubin R, Weaver E. Diffuse desmoplastic breast carcinoma metastases to the liver simulating cirrhosis at MR imaging: report of two cases. Radiology. 2001;221:117-121.

27. Chandrakar V, Isaacs $\mathrm{C}$. Breast cancer-related pseudocirrhosis and esophageal varices. Breast $\mathrm{J}$. 2005;11:301-302.

28. Sass DA, Clark K, Grzybicki D, Rabinovitz M, Shaw-Stiffel TA. Diffuse desmoplastic metastatic breast cancer simulating cirrhosis with severe portal hypertension: a case of "pseudocirrhosis". Dig Dis Sci. 2007;52:749-752.

29. Fournier C, Tisman G, Kleinman R, Park Y, Macdonald WD. Clinical evidence for overcoming capecitabine resistance in a woman with breast cancer terminating in radiologically occult micronodular pseudo-cirrhosis with portal hypertension: a case report. J Med Case Rep. 2010;4:112.

30. Leyden JE, Looby S, Gulmann C, Patchett SE. Cryptogenic cirrhosis: or is it? An unusual case of portal hypertension. Gut. 2010;59:1206.

31. Jüngst C, Krämer J, Schneider G, Lammert F, Zimmer V. Subacute liver failure by pseudocirrhotic metastatic breast cancer infiltration. Ann Hepatol. 2013;12:834-836.

32. Lee SL, Chang ED, Na SJ, Kim JS, An HJ, Ko YH, et al. Pseudocirrhosis of breast cancer metastases to the liver treated by chemotherapy.Cancer Res Treat. 2014;46:98-103.

33. Aoyagi T, Takabe K, Tamanuki T, Matsubara H, Matsuzaki H. Pseudocirrhosis after chemotherapy in breast cancer, case reports. Breast Cancer. 2018;25:614-618.

34. Diamond JR, Finlayson CA, Borges VF. Hepatic complications of breast cancer. Lancet Oncol. 2009;10:615-621.

\section{Tables}


Table 1. Characteristics of patients with breast cancer and pseudocirrhosis with and without portal hypertension

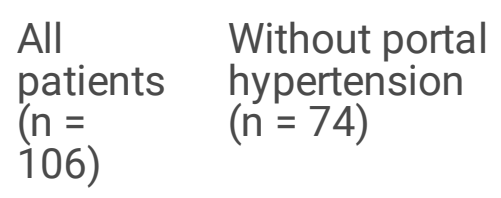

With portal hypertension $(\mathrm{n}=32)$

Age (years)

Median (range)

$54(32-\quad 54(35-79)$

79)

$>60, \mathrm{n}(\%)$

$23(22) \quad 19(26)$

$4(13)$

0.199

Breast cancer stage at diagnosis, $\mathrm{n}$

(\%)

I-III

IV

Pathology subtypes, n (\%)

HR+/HER2-

HER2+

Triple negative

Viral hepatitis, $\mathrm{n}(\%)$

HBsAg

\section{Anti-HCV}

Liver lobe with initial metastases

73 (69) $52(70)$

33 (31) $22(30)$

$21(66)$

$11(34)$

$53(32-69)$

0.645

(31) $22(30)$

$66(62)$

45 (61)

$32(30) \quad 23(31)$

8 (8)

$6(8)$

2 (6)

$21(66)$

$9(28)$

$9(8) \quad 6(8)$

3 (9)

0.569

0.882

\section{Left}

Right

Right and left

Number of initial liver metastases

$\leq 3$
4
$\geq 5$
$\begin{aligned} & \text { Concurrent visceral metastases, } n \\ & (\%)\end{aligned}$

(\%)

Lung

5 (5) $3(4)$

20 (19) $15(20)$

81 (76) $\quad 56(76)$

25 (78)

$2(6)$

$5(16)$

0.778 


\begin{tabular}{|lllll|}
\hline Brain & $23(22)$ & $19(26)$ & $4(13)$ & 0.239 \\
\hline $\begin{array}{l}\text { Months from Stage IV to } \\
\text { pseudocirrhosis (range) }\end{array}$ & $27(0-$ & $26(2-85)$ & $34(0-140)$ & 0.315 \\
\hline $\begin{array}{l}\text { Abbreviations: HR, hormone receptor; HER2, human epithelial growth factor receptor type 2; HBsAg: } \\
\text { hepatitis B virus surface antigen; anti-HCV: hepatitis C virus antibody. }\end{array}$ \\
\hline
\end{tabular}


Table 2. Systemic chemotherapy regimens and complications of portal hypertension after pseudocirrhosis diagnosis in patients with breast cancer

$\begin{array}{llll}\text { All } & \text { Without portal } & \text { With portal } & p \\ \text { patients } & \begin{array}{l}\text { hypertension } \\ (n=\end{array} & \begin{array}{l}\text { hypertension } \\ (n=74)\end{array} & \text { value } \\ 106) & & (n=32) & \end{array}$

Systemic chemotherapy

regimens, $\mathrm{n}(\%)$

$\begin{array}{lllll}\text { Taxane } & 55(52) & 45(61) & 10(31) & \mathbf{0 . 0 0 5} \\ \text { Doxorubicin } & 24(23) & 21(28) & 3(9) & \mathbf{0 . 0 4 2} \\ \text { Fluorouracil } & 60(57) & 39(52) & 21(66) & 0.287 \\ \text { Platinum } & 49(46) & 34(46) & 15(47) & 1.000\end{array}$

Complications of portal

hypertension, $\mathrm{n}(\%)$

\begin{tabular}{lcccc|}
$\begin{array}{l}\text { Gastrointestinal bleeding } \\
\text { episode }\end{array}$ & $31(29)$ & $6(8)$ & $23(72)$ & $<0.001$ \\
\hline Thrombocytopenia $^{\mathrm{a}}$ & $70(66)$ & $41(55)$ & $29(91)$ & $<0.001$ \\
\hline Hyperbilirubinemia $^{\mathrm{b}}$ & $64(60)$ & $36(49)$ & $28(88)$ & $<0.001$ \\
\hline Coagulopathy $^{\mathrm{c}}$ & $37(35)$ & $14(19)$ & $23(72)$ & $<0.001$
\end{tabular}

Hyperammonemia ${ }^{d}$

Received transfusion

$49(46) \quad 22(30)$

$27(84)$

$<0.001$

Abbreviations: HR, hormone receptor; HER2, human epithelial growth factor receptor type 2

${ }^{\text {aP }}$ latelet count $<100,000 / \mu \mathrm{L}$.

${ }^{\mathrm{b}}$ Total bilirubin $>1.5$ times the upper limit of normal.

'Blood International normalized ratio $>1.5$ times the upper limit of normal.

${ }^{\mathrm{d}}$ Blood ammonia $>50 \mu \mathrm{mol} / \mathrm{L}$. 
Table 3. Univariate and multivariate analyses of overall survival in patients with pseudocirrhosis

Variables Hazard ratio $(95 \% \mathrm{Cl})$

pvalue

\section{Univariate analysis}

With vs. without portal hypertension

$2.16(1.33-3.53)$

0.002

Age $>60$ vs. $\leq 60$ years

$1.17(0.67-2.04)$

0.592

Pathology subtypes

HR+/HER2-

Ref.

HER2+

$0.51(0.29-0.88)$

0.015

Triple negative

$2.91(1.19-7.08)$

0.019

Breast cancer stage at diagnosis

I-III

Ref.

IV

$1.13(0.69-1.86)$

0.616

Visceral organ metastases

Lung vs. non lung

$1.00(0.63-1.61)$

0.988

Brain vs. non brain

$1.83(0.99-3.38)$

0.054

\section{Multivariate analysis}

With vs. without portal hypertension

$2.09(1.22-3.57)$

0.007

Age $>60$ vs. $\leq 60$ years

$1.01(0.57-1.79)$

0.964

Pathology subtypes

HR+/HER2-

Ref.

HER-2+

$0.60(0.31-1.15)$

0.121

Triple negative

$3.15(1.27-7.81)$

0.013

Breast cancer stage at diagnosis

|- III

Ref.

IV

$1.26(0.74-2.15)$

0.393

Visceral organ metastases

Lung vs. non lung

$1.09(0.64-1.86)$

0.746

Brain vs. non brain

$0.78(0.39-1.51)$

0.439

Abbreviations: HR, hormone receptor; HER2, human epithelial growth factor receptor type 2; Cl, confidence interval. 
Table 4. Summary of outcomes of patients with pseudocirrhosis and esophageal or gastric varices

$\begin{array}{lllll}\text { Study [reference] } & \begin{array}{l}\text { Study } \\ \text { years } \\ \text { range }\end{array} & \begin{array}{l}\text { Patients } \\ \text { numbers }\end{array} & \begin{array}{l}\text { Pathology subtypes of breast } \\ \text { cancer (number) }\end{array} & \begin{array}{l}\text { Status, after } \\ \text { months }(\mathrm{m})\end{array} \\ & & & \end{array}$

Young, et al,

2

Unknown

Unknown

1994[13]

$\begin{array}{lllll}\text { Sonnenblick et } & 2006- & 1 & \text { Unknown } & \text { Unknown } \\ \text { al, 2011 [15] } & 2009 & & \end{array}$

Adike et al,

2

2016[16]

$2 \quad$ HR+/HER2-

Died, $1 \mathrm{~m}$

HER2+

Alive, $18 \mathrm{~m}$

Engelman, et al, $\quad 2003$

11

Unknown

Unknown

2020[18]

Nascimento et al,

2001 [26]

Chandrakar et al, 2002

2005 [27]

Sass et al,

2007[28]

Fournier et al,

2010 [29]

Leyden et al,

2010[30]

Jüngst et al,

2013[31]

Lee et al,

2014[32]

Aoyagi et al,

2018 [33]

$\begin{array}{ll}\text { Present study } & 2005- \\ & 2017\end{array}$

2008

2012
2

Unknown

Died, $1 \mathrm{~m}$

HER2+

Unknown

HR+/HER2-

Died

1

1 HR+/HER2-

Hospice

1

HR+/HER2-

Died, $1 \mathrm{~m}$

$1 \quad$ HR+/HER2-

Died, $1 \mathrm{~m}$

1 HER2+

Died

1

HR+/HER2-

Hospice

$H R+/ H E R 2-(n=21)$

HER2 + $(n=9)$

Median OS: $5 \mathrm{~m}$

(0.2-32) $\mathrm{m}$

Triple negative $(n=2)$

Abbreviations: HR, hormone receptor; HER-2, human epithelial growth factor receptor type 2; OS, overall survival. 


\section{Figures}
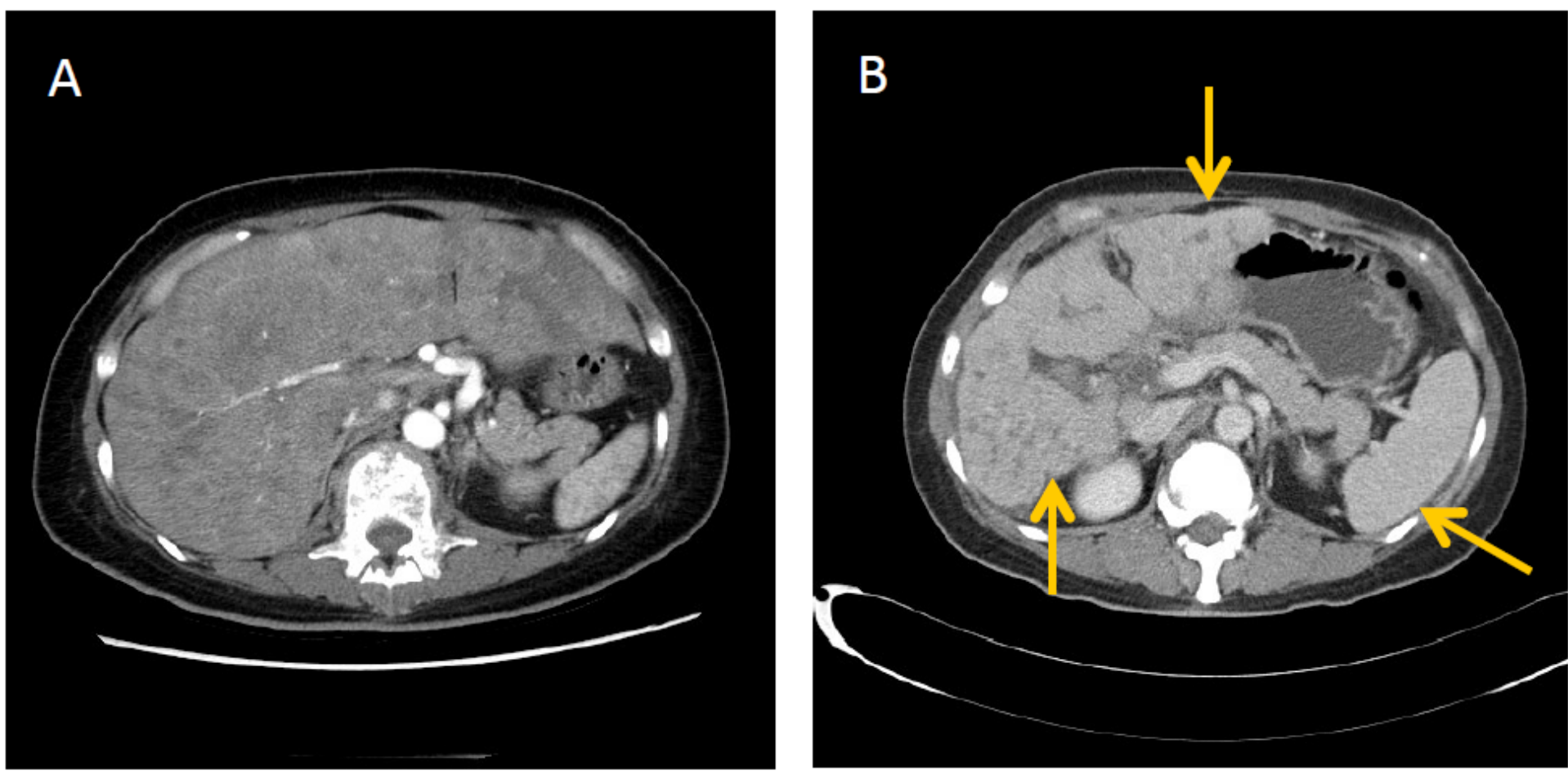

\section{Figure 1}

Computed tomography abdomen scan with contrast showing (A) diffuse liver metastases before treatment and (B) pseudocirrhosis with irregular hepatic contours, segmental volume loss, and splenomegaly (arrows) after systemic treatment for 2 years. 


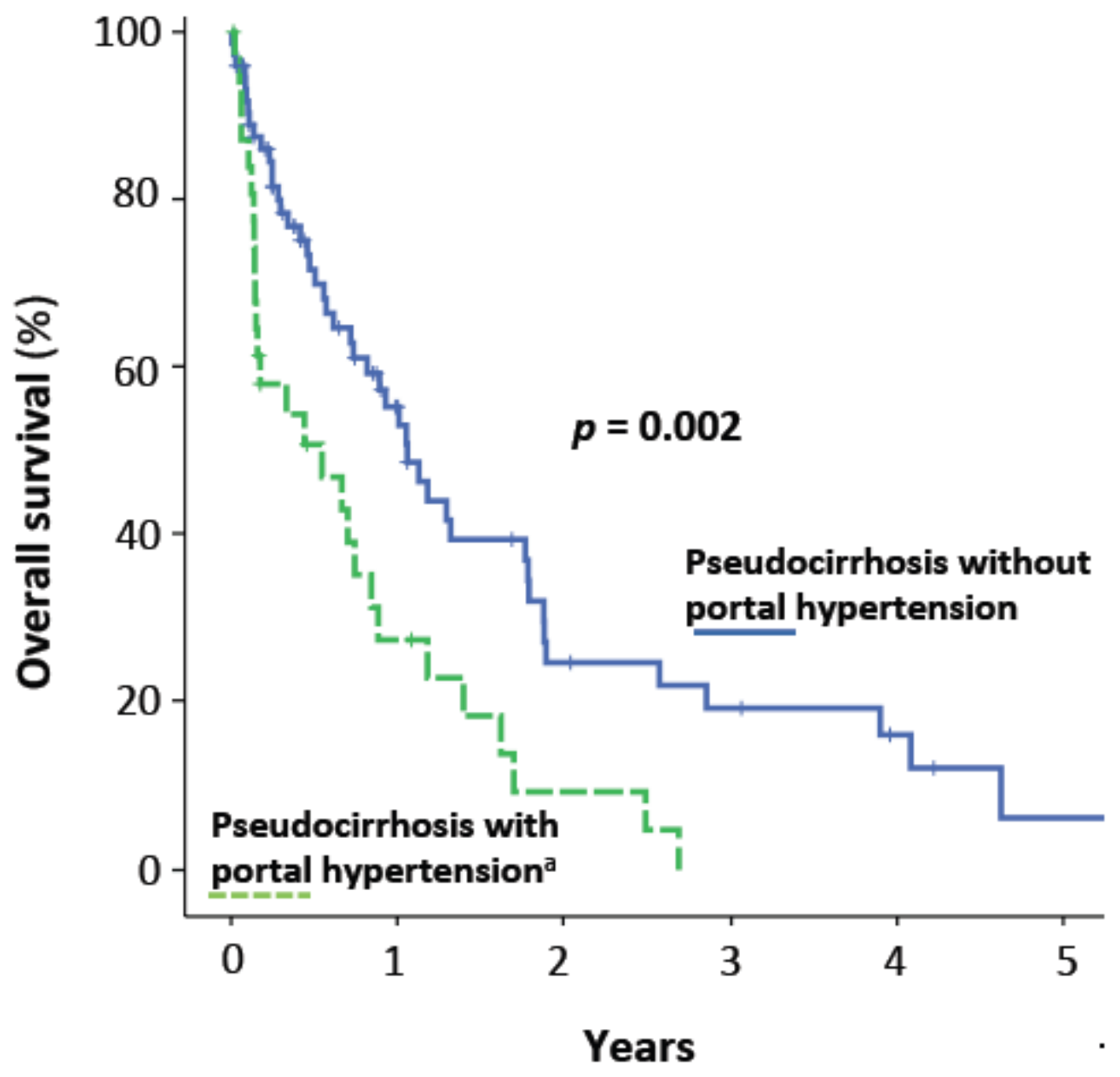

\begin{tabular}{r|rrrrrr} 
Numbers at risk & \multicolumn{7}{|c}{} \\
\hline Without portal hypertension $^{7}$ & 74 & 26 & 10 & 7 & 4 & 1 \\
With portal hypertension $^{\mathrm{a}}$ & 32 & 7 & 2 & 0 & 0 & 0
\end{tabular}

Figure 2

OS of patients with metastatic breast cancer and pseudocirrhosis with and without portal hypertensiona. The median OS was 5 months in patients with portal hypertension and 13 months in those without $(\mathrm{p}=$ .002). OS: overall survival. aEvident esophageal or gastric varices on esophagogastroduodenoscopy. 


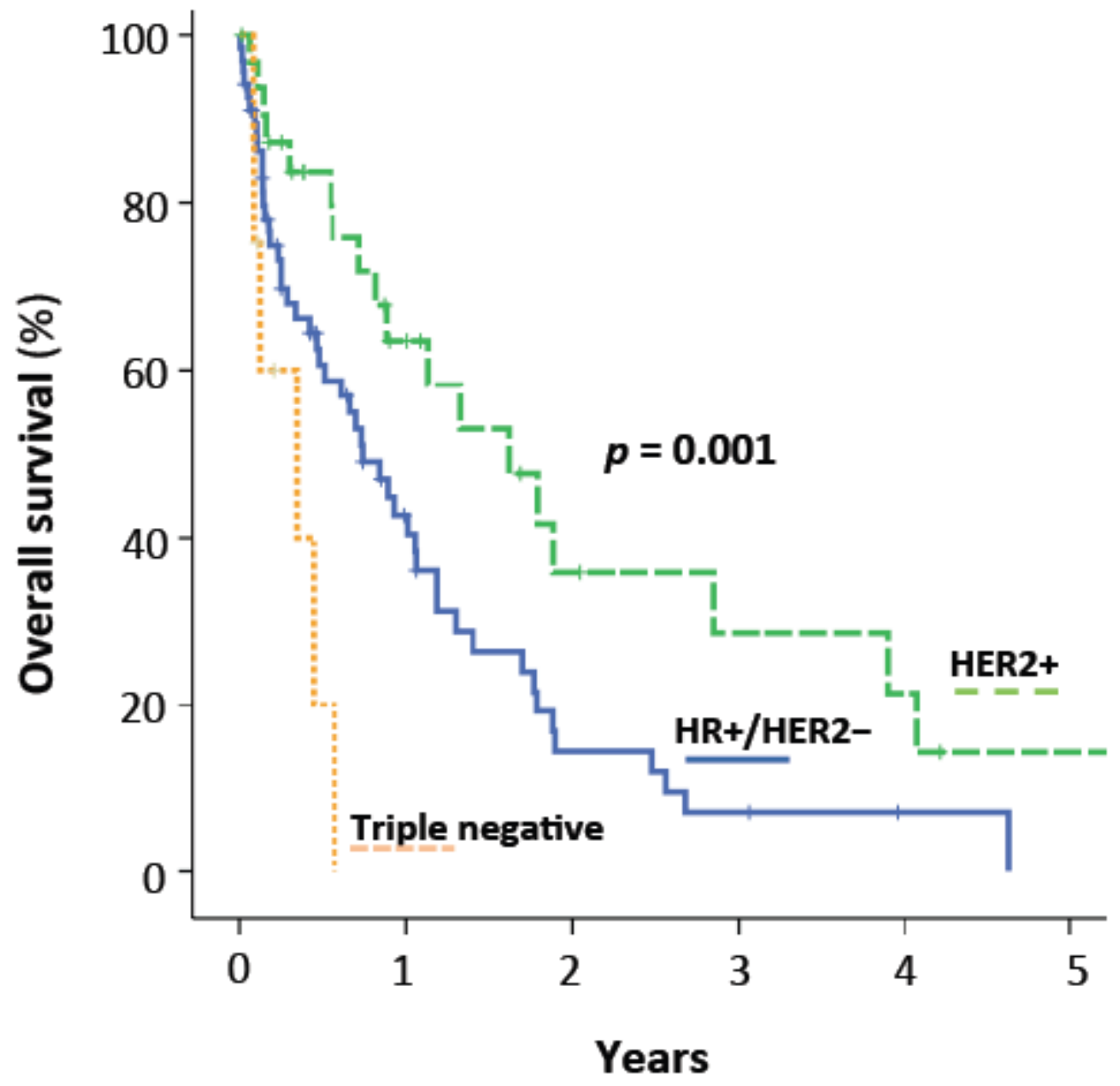

\begin{tabular}{r|rrrrrr} 
Numbers at risk & \multicolumn{7}{|c}{} \\
HER2+ & 32 & 14 & 5 & 4 & 3 & 1 \\
HR+/HER2- & 66 & 19 & 6 & 3 & 1 & 0 \\
Triple negative & 8 & 0 & 0 & 0 & 0 & 0
\end{tabular}

Figure 3

OS of patients with metastatic breast cancer and pseudocirrhosis across HER2+, HR+/HER2-, and triplenegative subtypes. Median OS was 16,9 , and 2 months, respectively $(p=.002)$. OS: overall survival; HR, hormone receptor; HER2, human epithelial growth factor receptor type 2. 

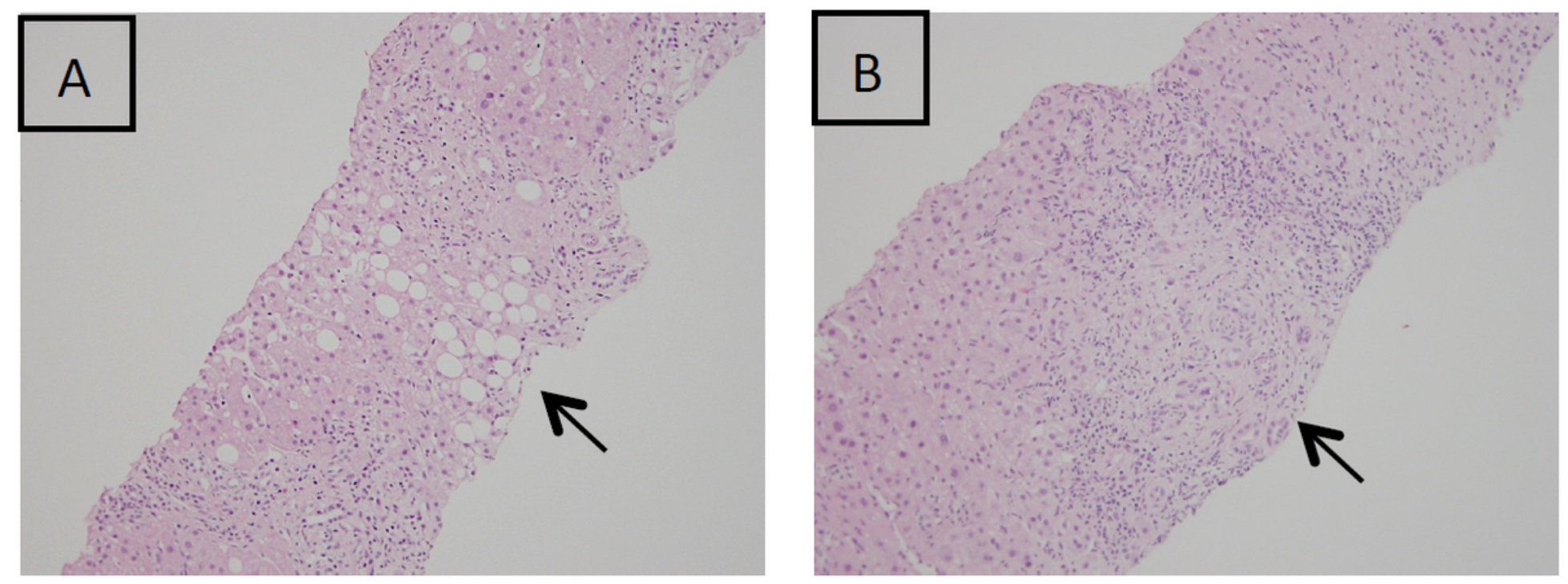

\section{Figure 4}

Microscopic findings of pseudocirrhosis through hematoxylin and eosin staining, 100x. (A) Fatty change in liver parenchyma. (B) Fibrosis at the portal area.

\section{Supplementary Files}

This is a list of supplementary files associated with this preprint. Click to download.

- 20211031 supplementarytable.docx 